[L. M. L. Cantu, J. Grohmann, W. Meier, M. Aigner, Temperature measurement in confined swirling spray flames by vibrational anti-Stokes Raman spectroscopy, Experimental Thermal and Fluid Science, Volume 95 (2018), 52-59]

The original publication is available at https://www.sciencedirect.com/science/article/pii/S089417771830061X

https://doi.org/10.1016/j.expthermflusci.2018.01.029

(C) <2018>. This manuscript version is made available under the CC-BY-NC-ND 4.0 license http://creativecommons.org/licenses/by-nc-nd/4.0/ 


\title{
TEMPERATURE MEASUREMENTS IN CONFINED SWIRLING SPRAY FLAMES BY VIBRATIONAL COHERENT ANTI- STOKES RAMAN SPECTROSCOPY
}

\author{
Luca M. L. Cantu*, Jasper Grohmann", Wolfgang Meier ${ }^{\dagger}$ and Manfred Aigner* \\ luca.cantu@dlr.de \\ Institute of Combustion Technology \\ German Aerospace Center (DLR) \\ Pfaffenwaldring 38-40, 70569 Stuttgart, Germany
}

\begin{abstract}
A gas turbine model combustor for swirling spray flames has been operated at atmospheric pressure with n-hexane, n-dodecane and kerosene Jet A-1. Temperature measurements were performed using single-shot broadband shifted vibrational coherent anti-Stokes Raman spectroscopy (SV-CARS). Series of 1200 single-shot measurements were performed at different radial and vertical locations in the flames from which the temperature distributions were deduced. In regions with high droplet load a significant number of CARS spectra were discarded due to large signal background from laser-induced breakdown effects. Results from the flames burning different fuels were compared and revealed considerable differences in the temperature profiles. The temperature measurements are part of a comprehensive research program that aims at the design of alternative fuels for aero engines and stationary gas turbines. In addition to the experimental characterization of the spray flames, the datasets are used for the validation and improvement of computational models.
\end{abstract}

Keywords: alternative jet fuels, spray flame, CARS, temperature measurements

\section{Introduction}

The design and production process of alternative jet fuels are an active field of research due to ecological reasons, limited resources and concerns of import dependency [1,2]. The main focus is towards drop-in fuels which can be used without modifications of the engine or penalizations in terms of performances and safety [3]. Understanding the influence of the fuel composition on the combustion behavior offers the opportunity for more suitable blend formulations which could reduce emissions and provide more efficient combustion performance [4]. Here, validated numerical models are the key step to improve design tools. However, the effects of individual hydrocarbons, hydrocarbon groups, and their ratio in blends on combustion processes are not fully understood. In particular, well documented test cases and comprehensive experimental data sets are needed to improve the understanding. To achieve this goal, a model combustor was designed [5] at the German Aerospace Center (DLR) to simulate key features of a real aeroengine combustor: air-blast atomization of liquid fuel and a turbulent swirling flow field inside a confined combustion chamber. In the current work, single-component fuels were tested to reduce +Experiments were performed with two fuels from the chemical class of linear hydrocarbons (nhexane and n-dodecane). For comparison, kerosene Jet A-1 was used as technical reference. The burner was operated at ambient pressure. This provided simplified conditions to validate

\footnotetext{
${ }^{*}$ Research Associate, DLR, Institute of Combustion Technology, Stuttgart, Germany

${ }^{\dagger}$ Senior Scientist, DLR, Institute of Combustion Technology, Stuttgart, Germany

* Professor, DLR, Director of the Institute of Combustion Technology, Stuttgart, Germany
} 
numerical models [6] in order to gain a fundamental understanding of the influence of singlecomponent fuels before working at realistic high pressure conditions and complex fuel mixtures. The capability of the burner to provide optical access from multiple sides makes it particularly suitable for non-intrusive laser diagnostics techniques. Stereo particle image velocimetry (SPIV), $\mathrm{CH}^{*}$ chemiluminescence imaging, Mie scattering off fuel droplets, and phase Doppler anemometry (PDA) were already employed in previous measurement campaigns [5,7,8]. However, for modelling and validating combustion process simulations, reliable temperature measurements are essential. Among non-intrusive (laser-based) techniques for combustion diagnostics, coherent anti-Stokes Raman spectroscopy (CARS) [9,10] has long been established as a temperature measuring tool in numerous combustion environments. CARS is a non-linear spectroscopic technique that provides spatially and temporally resolved temperatures and species concentrations by probing molecular Raman shifts. Three coherent laser beams (pump, Stokes and probe) are focused and crossed in the region of interest generating a CARS signal beam as shown in Figure 1a. The frequencies of the three beams are chosen such that their interaction excites the molecular vibrational transitions of the $\mathrm{N}_{2}$ Q-branch. In broadband vibrational CARS the probe beam has a broad bandwidth that covers several vibrational and rotational transitions of the molecule.
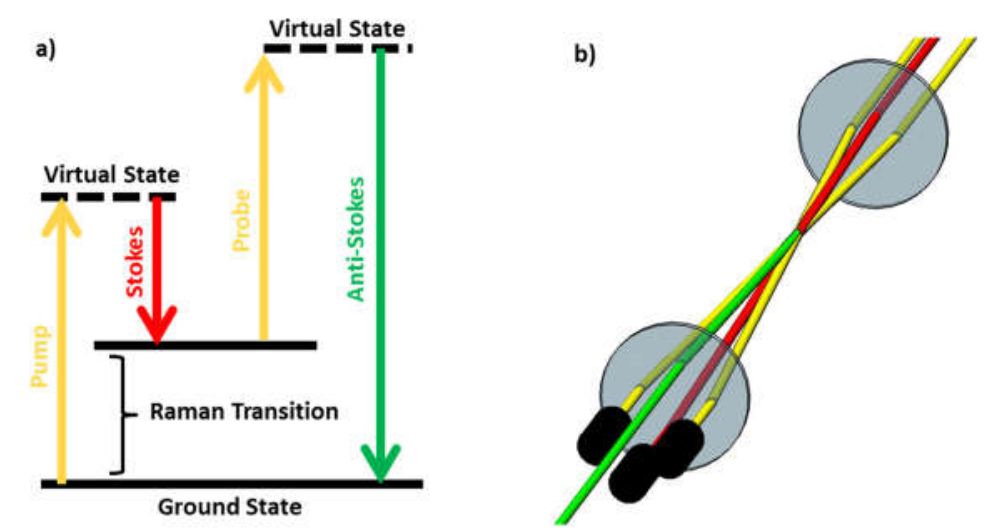

Figure 1. a) SV-CARS energy level diagram; b) folded BOXCARS phase matching configuration.

The resulting signal beam carries the Raman spectra of $\mathrm{N}_{2}$. Processing of the spectra allows temperature to be obtained by fitting the spectral shape. In broadband vibrational CARS, a spectrum is obtained with each single laser shot and the temperature distribution (probability density function, pdf) is obtained from the measurement of a large number of single spectra. The temperature pdfs carry important information about the combustion process. They reflect the reaction progress, quantify effects of heat loss and are an important quantity for analyzing NO formation. An additional aspect addressed in this paper is to analyze and understand the influence of different fuels on the temperature distribution. To support the interpretation, also results from previous measurements in the same burner are taken into consideration.

\section{Experimental setup}




\subsection{Facility}

The description of the gas turbine model combustor and the corresponding facility and fuel supply system has already been described in detail by Grohmann et al. [5]. Hence, a minimal description of the facility will be provided. The nozzle of the burner, shown in Figure 2a, consisted of two co-axial, co-rotating swirlers with a diameter of $8 \mathrm{~mm}$ (inner) and $11.6 \mathrm{~mm}$ (outer) respectively. An annular ring with a sharp edge separated the two air flows. A pressureswirl atomizer (Schlick 121) sprayed the fuel onto the inner surface. The thin liquid film formed was then re-atomized at the atomizer lip and then injected into the combustion chamber. The combustion chamber $(85 \times 85 \times 169 \mathrm{~mm})$ was equipped with four quartz windows with antireflective coating and provided full optical access. The combustor was mounted on a three-axis translation stage. The facility setup scheme is shown in Figure $2 \mathrm{~b}$. A compressor supplied dry air which was preheated by an electric heater $(\sim 6 \mathrm{~kW})$ before being sent to the combustor; a thermal mass flow controller (Bronkhorst EL-FLOW select F-203AV, accuracy $\pm 0.1 \%$ full scale) regulated the air mass flow rate. Fuel was pressurized inside a steel cylinder and sent to the burner through a mass flow controller (Bronkhorst mini CORI-FLOW M14, accuracy $\pm 0.2 \%$ full scale). The air temperature was measured by a thermocouple inside the plenum and kept constant at $323 \mathrm{~K}$ : this value was chosen to provide a stable and repeatable boundary condition. The fuel temperature was measured upstream of the first atomization and kept constant at $303 \mathrm{~K}$. All tests were performed at a global equivalence ratio $\Phi=0.8$ and at atmospheric pressure.
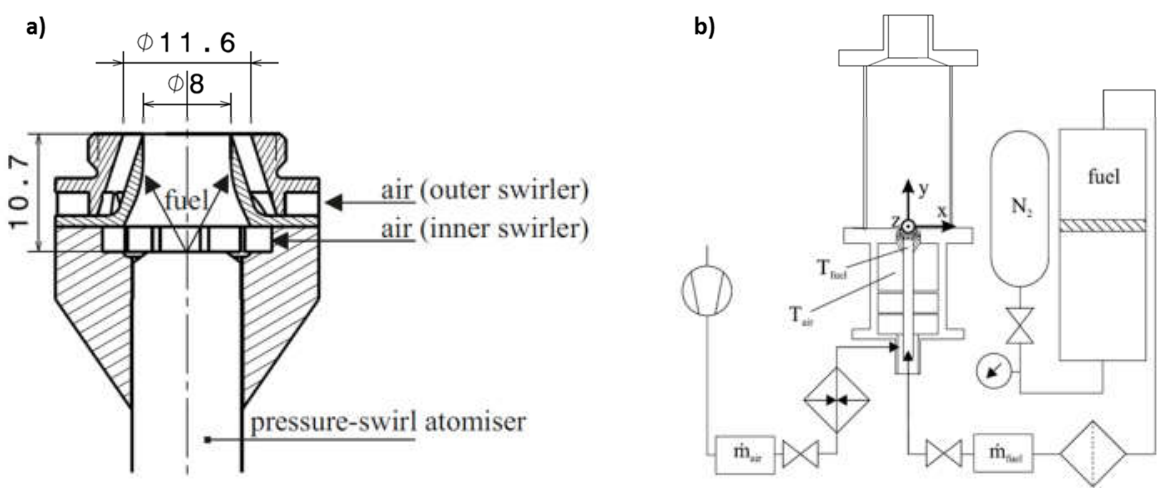

Figure 2. a) nozzle; b) rig (not in scale).

\subsection{Optical setup}

A frequency doubled Nd:YAG laser (Quanta Ray Pro 290) with a repetition rate of $10 \mathrm{~Hz}(\lambda=$ $532 \mathrm{~nm}$, pulse duration $\sim 8$ ns) was used to pump two dye lasers (Sirah Double Dye PrecisonScan): a narrow-band dye laser was tuned to produce $591 \mathrm{~nm}$ yellow light (dye: kiton red in ethanol) and provided the pump and probe beams while the broad-band dye laser (Stokes beam) was set to produce peak energy at $685 \mathrm{~nm}$ red light (dye: pyridine 1 in ethanol). Compared to the often used wavelengths of $532 \mathrm{~nm}$ and $607 \mathrm{~nm}$, these wavelengths are longer in order to shift the CARS signal from $473 \mathrm{~nm}$ to $519 \mathrm{~nm}$. This arrangement avoids interference due to laserinduced $\mathrm{C}_{2}$ emissions [11]. The lasers and the related optical components were mounted in a mobile container previously described by Geigle et al. [12]. Outside the laser container the three beams (two narrow-bands and one broad-band) were relayed by a series of high-reflectivity broad-band mirrors close to the measurement location as shown in Figure 3. A $250 \mathrm{~mm}$ focal length (FL) lens focused the three beams to the probe volume in the center-plane of the swirl burner. A removable 50:50 beam splitter was placed in front of the combustor and deflected the 
beams to a focal plane imaging (FPI) camera (Data Ray WinCamD). The camera was placed at the focusing plane of the three beams and was used to perform a fine alignment of the crossing point. The beam splitter was removed for the measurements in the combustor. Spatial resolution was measured before each experiment and it was in the average $\mathrm{L}_{95 \%} \sim 2.2 \mathrm{~mm}$ in the beam direction and $\leq 0.1 \mathrm{~mm}$ in diameter. To generate a high-intensity and coherent signal beam, constructive laser beam interference is required at the measurement point, where the three laser beams are crossed. In this experiment, a folded BOXCARS [9] configuration was used to achieve phase matching as shown in Figure 1b.

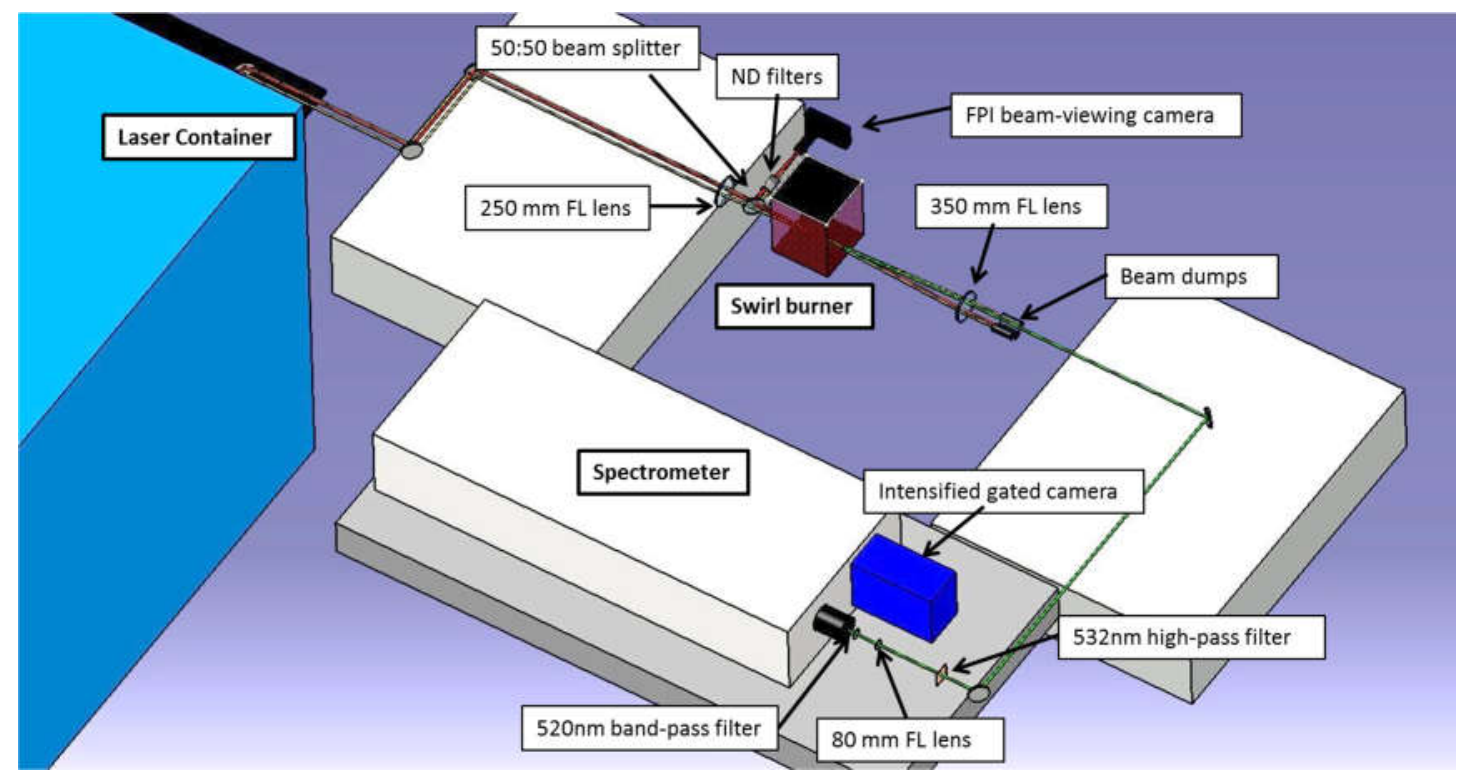

Figure 3. Optical setup.

At the crossing point, the $519 \mathrm{~nm}$ (green light) CARS anti-Stokes signal was generated and propagated laser-like in forward direction. It was collimated by a $350 \mathrm{~mm}$ FL lens and sent to the spectrometer by a series of dichroic mirrors. The three signal generating laser beams were blocked by beam dumps placed behind the collimating lens. An $80 \mathrm{~mm}$ FL lens focused the signal to the $50 \mu \mathrm{m}$ wide entrance slit of a spectrometer (ISA Instruments THR 1000) equipped with a 1200 groves/mm diffraction grating. Two filters, a $532 \mathrm{~nm}$ high-pass filter (Linos C-54) and a $520 \mathrm{~nm}$ centered band-pass filter (Chroma ET529/20m, transmission 98\% at $520 \mathrm{~nm}$ ) were placed in front of the spectrometer to eliminate residual stray light. Finally the dispersed CARS spectra were acquired by an intensified charge-coupled device (ICCD) camera (LaVision FlameStar2, resolution 1.21 pixels/wavenumber) placed at the end of the spectrometer.

\subsection{Data Analysis}

Measurements were performed at different axial and radial positions and at each measurement point 1200 spectra were acquired. Measurements were possible only in presence of $\mathrm{N}_{2}$ since the temperature was derived from the $\mathrm{N}_{2}$ spectrum. All measurements were processed to obtain CARS susceptibility spectra. The preprocessing consisted of subtracting an averaged background (recorded after each run) from each single shot taken. Then, all the single shot spectra were normalized by the non-resonant spectrum (average of two Argon spectra recorded before and after each experiment) to account for the intensity distribution of the broad-band dye laser $[13,14]$. Knowing the environmental conditions and chemical composition, theoretical spectra 
libraries were created. The spectral resolution of the CARS spectra depends on the instrument function which is predominantly dependent on the slit width of the spectrometer. It was determined from an averaged room temperature air spectrum which was recorded before each experiment and theoretically fitted. A DLR-developed fitting code was employed to iterate processed data and theoretical libraries until convergence was achieved. From the fitted spectral shape it was possible to determine the temperature. A filter was applied to discard poorly fitted data: based on the error sum of squares, fitted spectra were excluded from the statistical analysis whenever they exceeded an imposed value. Finally, statistical analysis was performed for each measurement point providing mean temperature $\left(\mathrm{T}_{\text {mean }}\right)$, standard deviation, most probable temperature $\left(\mathrm{T}_{\mathrm{mp}}\right), 5 \%$ and $95 \%$ confidence interval and temperature distributions. More details on the fitting code are provided by Lückerath et al. [15]. An example of processed spectra and algorithm output is shown in Figure 4.
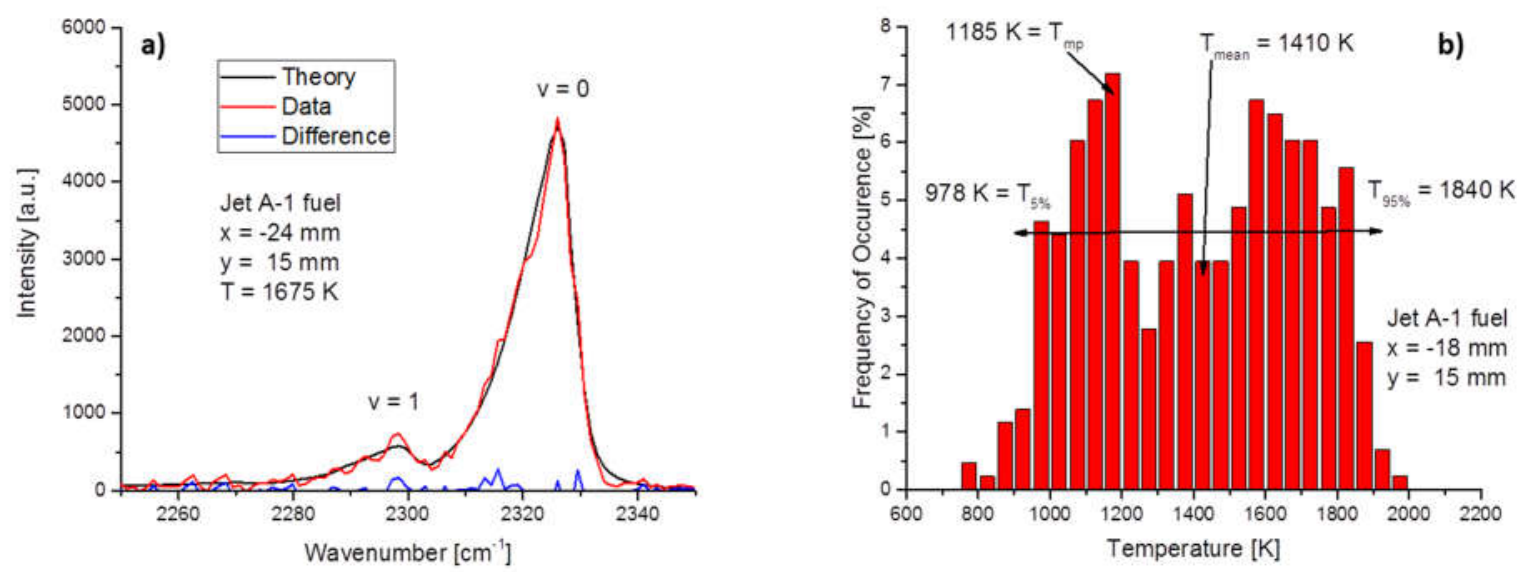

Figure 4. a) processed selected single shot spectrum; b) histogram showing temperature distribution of a selected single measurement point.

The temperature accuracy was estimated to be $\leq 4.5 \%$ by evaluating temperature fluctuations with respect to the mean in a methane-air flame over a McKenna burner. This value is also consistent with previous campaigns $[15,16]$ performed with the same system.

Before fitting the data, spectra affected by laser-induced optical breakdown [9] were discarded, thereby reducing the number of processed spectra: this effect was determined to be present if the baseline of a spectrum was higher than an imposed threshold. Laser-induced breakdown was observed in several measurements where the fuel was still in liquid phase: droplets acted as tiny lenses and focused the lasers causing electric field amplification. When plasma was formed, the medium was altered and precluded the measurement. As shown in Figure 5, the number of processed spectra varied by measurement location (a) and fuel properties (b). All measurements where collected at negative radial position $\mathrm{x}$ to be consistent with previous publications $[5,7,8]$. Figure 5a shows the percentage of successfully processed Jet-A1 fuel measurements at different locations in the flame. It can be noticed that closer to the injection at $\mathrm{y}=15 \mathrm{~mm}$ the number of single shots analyzed decreased due to the higher liquid load present in the flow (see Figure 9). As the fuel started burning and the liquid load decreased, the number of successful measurements increased. The same behavior is observed in the measurements further downstream ( $\mathrm{y}=25 \mathrm{~mm}$ and $\mathrm{y}=35 \mathrm{~mm}$ ): as the fuel was consumed, the number of processed single shots increased. The same trend was noticed for n-hexane and n-dodecane fuels (not shown). 

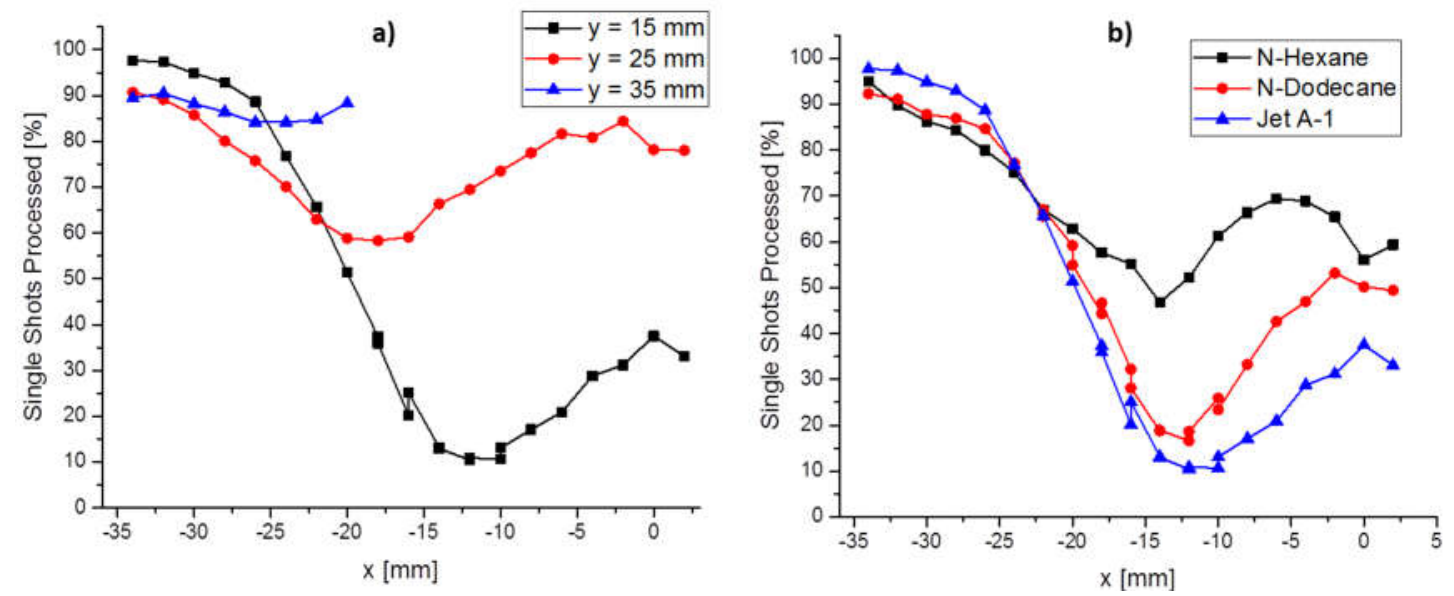

Figure 5. a) Jet A-1 single shots processed at different heights above burner surface; b) single shots processed for different fuels at $\mathrm{y}=15 \mathrm{~mm}$ above burner surface.

Figure $5 \mathrm{~b}$ shows the percentage of successfully processed single shots for different fuels at the same axial position of $y=15 \mathrm{~mm}$. It can be noticed that $\mathrm{n}$-hexane (lighter fuel) has more processed single shots in the injection region: this was due to smaller drops and a higher evaporation rate which led to a significant reduction of the liquid load. Contrary, n-dodecane and Jet-A1 (heavier fuels) showed higher liquid load close to the injection causing a higher number of rejected spectra. Laser-induced breakdown occurred only at high liquid loading in the flow. In these regions the temperatures are relatively low. Therefore, the evaluated temperature distribution may be biased towards to high values. This effect must be kept in mind when comparing (and/or validating) to numerical models. An assessment of the temperature bias due to the filtering is difficult and has not been investigated in detail so far.

\section{Results and Discussion}

Two fuels from the chemical class of linear hydrocarbons were tested in the gas turbine model combustor at an equivalence ratio of $\Phi=0.8$. Conventional kerosene Jet A-1 was used as a technical reference. The properties of the tested fuels and results from previous measurement campaigns $[5,7,8]$ at reference conditions are summarized in Table 1.

\begin{tabular}{|c|c|c|c|c|c|c|}
\hline$\Phi=0.8$ & $\mathbf{P}_{\text {th }}[\mathbf{k W}]$ & $\dot{\mathbf{m}}_{\text {fuel }}[\mathrm{g} / \mathrm{h}]$ & $\mathbf{T}_{\mathrm{ad}}[\mathbf{K}]$ & $\mathbf{T}_{\text {boil }}[\mathbf{K}]$ & $\begin{array}{l}\mathbf{d}_{\mathrm{drop}}[\mu \mathrm{m}] \\
(-20 ; 25)\end{array}$ & $\begin{array}{c}\text { Cond. liquid phase } \\
\text { load. [\%]@ }(-20 ; 25)\end{array}$ \\
\hline $\begin{array}{c}\text { n-hexane } \\
\left(\mathrm{C}_{6} \mathrm{H}_{14}\right)\end{array}$ & 10.116 & 814.1 & 2050 & 342 & 27.0 & 1.0 \\
\hline $\begin{array}{c}\text { n-dodecane } \\
\left(\mathrm{C}_{12} \mathrm{H}_{26}\right)\end{array}$ & 10.125 & 826.3 & 2055 & 490 & 38.0 & 6.3 \\
\hline Jet A-1 & 10.200 & 850.0 & 2065 & 449 & 36.5 & 5.4 \\
\hline
\end{tabular}

Table 1. Summary of tested fuels and respective properties at $\Phi=0.8$ : thermal power, fuel mass flow rate, adiabatic flame temperature, boiling temperature, droplet diameter and conditional liquid phase loading measured at $\mathrm{x}=-20 \mathrm{~mm}$ and $\mathrm{y}=25 \mathrm{~mm}$

For each fuel, three radial profiles (at y $=15,25$ and $35 \mathrm{~mm}$ respectively) were measured. Measurements were collected every $2 \mathrm{~mm}$ starting from the centerline: due to the symmetry of 
the burner, only half of the flame was considered. Each measurement point consisted of 1200 single shots that were individually processed.

\subsection{Temperature distribution}

Figure 6 shows (a) the mean temperature $\left(T_{\text {mean }}\right)$ and (b) the most probable temperature $\left(T_{m p}\right)$ recorded for all the fuels at $y=25 \mathrm{~mm}$. The temperature profiles reflect the different regions of the flame. The relatively low values around $\mathrm{x}=15 \mathrm{~mm}$ correspond to the inflow region where mixtures of cold and burned gas are found in varying compositions. In the outer radial region the temperatures reach the highest values. From velocity measurements in these flows it is known that this region is the outer recirculation zone [5]. The inner recirculation zone that is formed around the flame axis is characterized by medium to high temperatures. Between the inflow and the recirculation zones shear layers are formed which are typically characterized by large fluctuations and gradients of velocities and temperatures. Compared to the $T_{\text {mean }}$ profiles the shear layers are even more pronounced in the profiles of the $\mathrm{T}_{\mathrm{mp}}$ (Figure $6 \mathrm{~b}$ ).
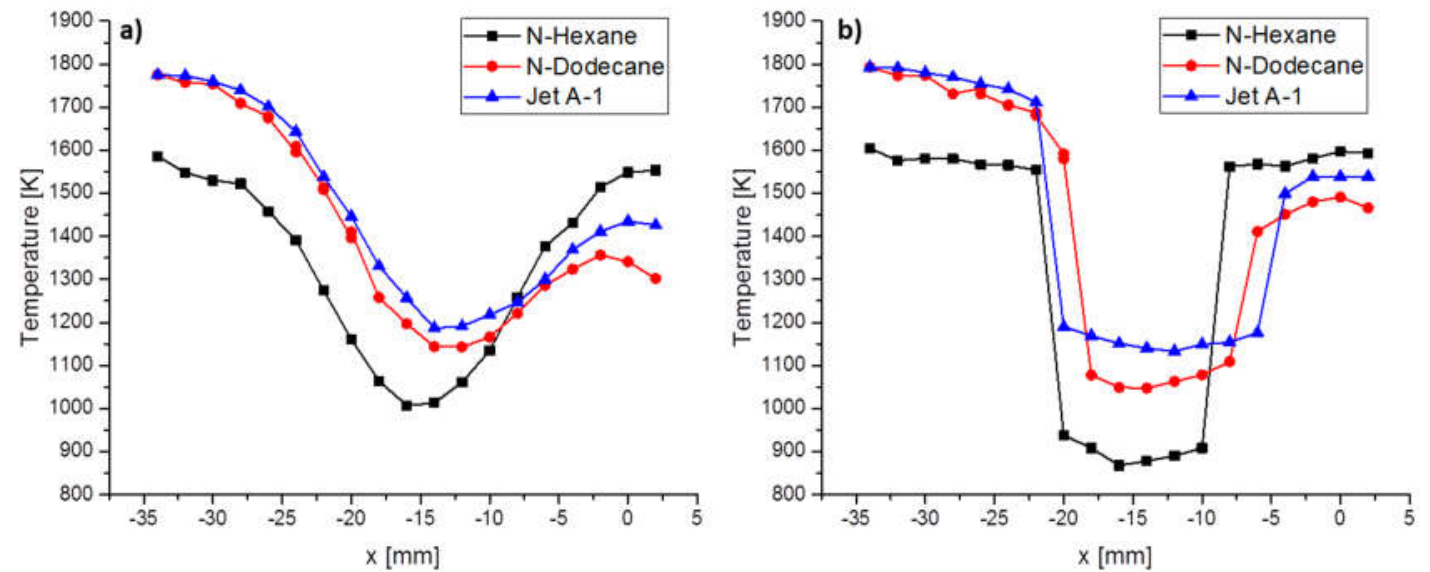

Figure 6. a) $\mathrm{T}_{\text {mean }}$ radial profile at $\mathrm{y}=25 \mathrm{~mm}$ for the tested fuels; b) $\mathrm{T}_{\mathrm{mp}}$ radial profile at $\mathrm{y}=25$ $\mathrm{mm}$ for the tested fuels.

The temperature profiles show that the Jet A-1 flame behaved very similar to the n-dodecane flame; this is in agreement with previous publications $[17,18]$ in which n-dodecane was suggested as a suitable surrogate fuel for jet engines. The maximum $T_{\text {mean }}$ and $T_{m p}$ are considerably below the adiabatic flame temperatures. This is explained by heat loss of hot combustion gases at the burner surface and the chamber walls, as well as by radiation. Compared to kerosene and n-dodecane, the temperature profile of the n-hexane flame is different with higher temperatures in the inner recirculation zone and lower temperatures in the outer recirculation zone. Because the adiabatic flame temperatures and thermal powers are very similar for all three flames, the differences are obviously due to different atomization and evaporation rates of the fuels which in turn influence the flame shape.

In regions with large turbulent fluctuations $T_{\text {mean }}$ and $T_{m p}$ do not yield a sufficient characterization of the thermal state of a flame. The variation of the thermal state can best be assessed from the temperature probability density functions (pdfs). Four examples from the nhexane flame are displayed in Figure 7 in the form of temperature histograms from different radial locations at the downstream location $y=25 \mathrm{~mm}$. At $\mathrm{x}=-34 \mathrm{~mm}$ the pdf is mono-modal and the relatively high temperature level indicates that mostly exhaust gas was present at this 
location. At lower radial positions the pdfs become bimodal with temperature maxima around $900 \mathrm{~K}$ and $1500 \mathrm{~K}$. The bimodality is evidence of an intermittent presence of partially reacted mixtures at medium temperatures and (almost) completely reacted exhaust gas. The intermittency can be caused by a fluctuating shear layer between inflowing and recirculating gas or by a flame front that crosses the measurement position randomly. Temperature distributions of similar shape have been reported from Raman measurements in gaseous swirl flames [19,20].
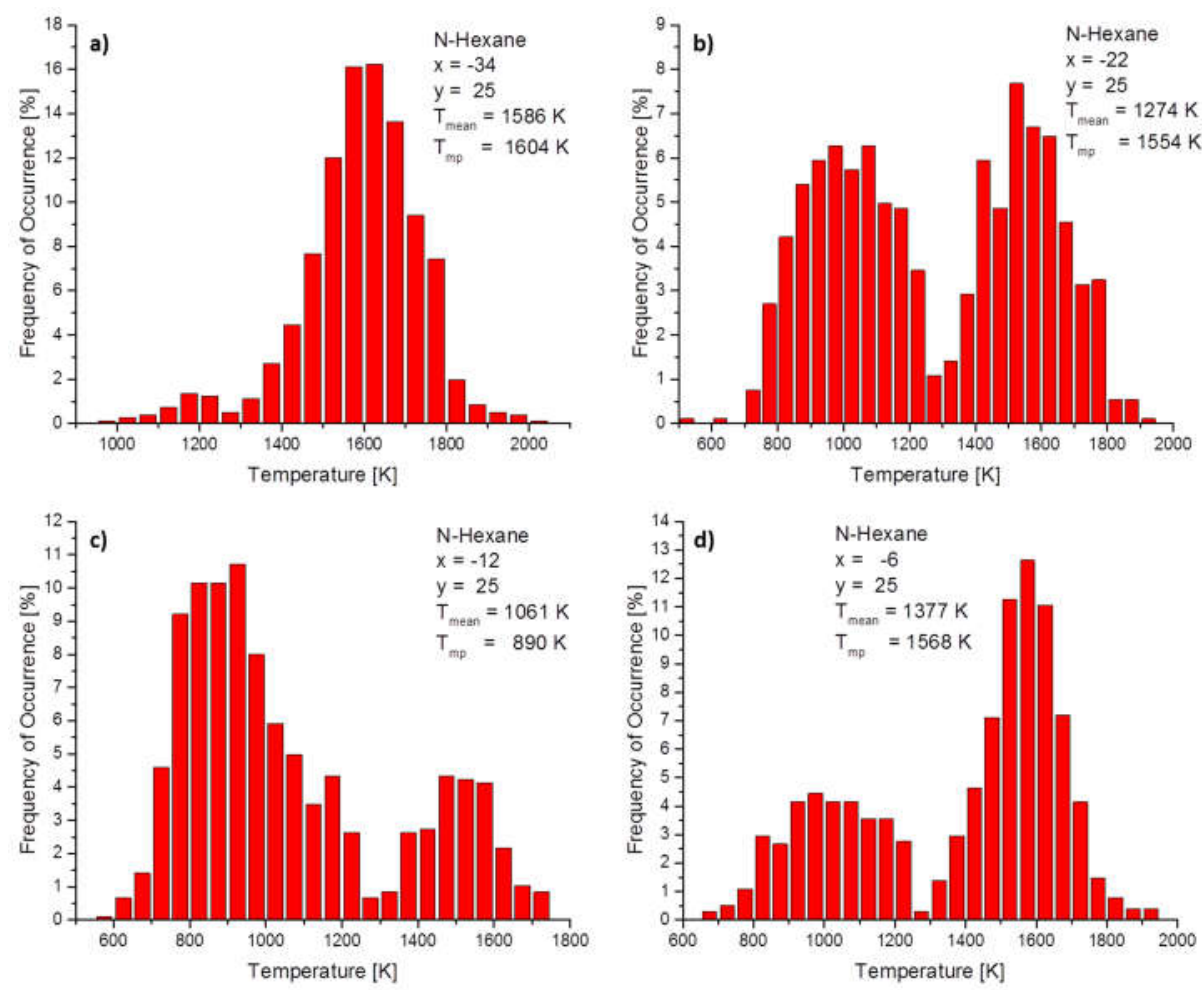

Figure 7. Histograms representing selected measurement points in n-hexane-air flame; a) single distribution; b) symmetric double distribution; c) asymmetric distribution towards cold temperatures; d) asymmetric distribution towards hot temperatures.

Because the presentation of temperature pdfs for all measurement positions is too space filling, the $5 \%$ and $95 \%$ confidence interval of the temperature distribution may be used to specify the width of the distribution. As an example, Figure 8 shows radial profiles of $\mathrm{T}_{\text {mean }}, \mathrm{T}_{\mathrm{mp}}$ and $\mathrm{T}_{5-95 \%}$ for the $\mathrm{n}$-hexane flame at $\mathrm{y}=25 \mathrm{~mm}$. It is seen that the width of the temperature distributions is particularly large near the inflow region. Interestingly, the most probable temperatures are nearly constant except for the jump at $\mathrm{x}=-10$ and $-20 \mathrm{~mm}$ where the maximum of the bimodal distributions switches from medium to high temperature values. 


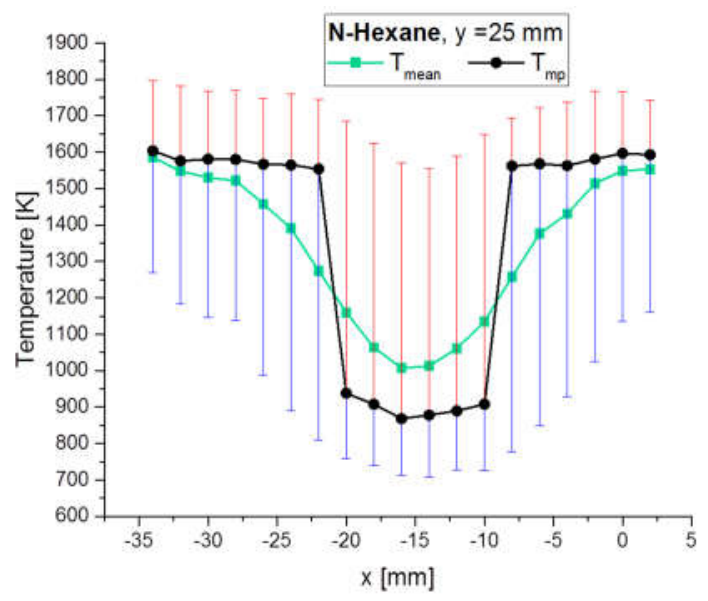

Figure 8. $T_{\text {mean }}$ and $T_{m p}$ profiles for $n-h e x a n e$ at $y=25 \mathrm{~mm} ; 5 \%$ and $95 \%$ temperature probability are shown as red and blue bar with respect to $\mathrm{T}_{\mathrm{mp}}$.

\subsection{Comparisons of the flames}

Figure 9 shows contour plots of $\mathrm{T}_{\text {mean }}$ for the $\mathrm{n}$-hexane and $\mathrm{n}$-dodecane flames. The contour plot was obtained by interpolating the temperatures from all measurement points (marked as black dots) using the software Origin 2016. Even though the measurement points were not evenly distributed over the displayed region, the interpolation provides a useful presentation for the comparison.
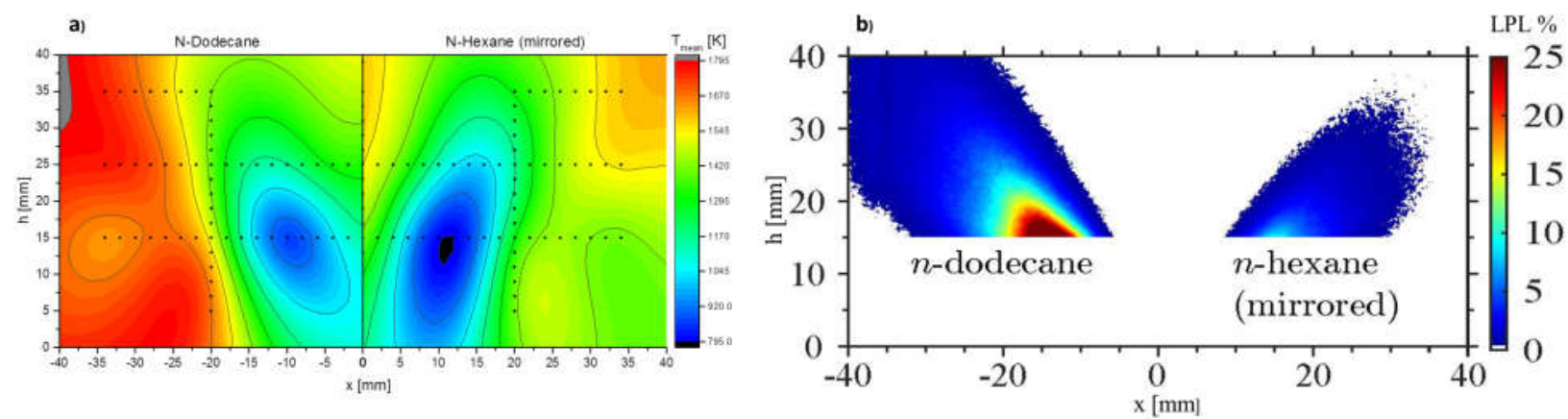

Figure 9. Comparison between a) CARS $T_{\text {mean }}$ measurements for $n$-dodecane and mirrored nhexane (black dots represent single CARS measurement points) and b) Mie scattering liquid phase loading (LPL) measurements [8].

It can be seen that the shapes of the temperature distributions differ and that the n-dodecane flame reaches a higher temperature level. As stated above, the reason for the differences lies in the different droplet sizes and evaporation rates. n-Hexane has a lower boiling point than $\mathrm{n}$ dodecane (see Table 1) and evaporates faster. The distribution of the liquid phase loading (Figure 9 b) reflects this property: the $\mathrm{n}$-hexane spray penetrates the combustion chamber up to $\mathrm{y} \approx 33$ $\mathrm{mm}$ whereas n-dodecane droplets reach up to $\mathrm{y} \approx 55 \mathrm{~mm}$.

Because temperature differences between the flames can hardly be explained by the liquid fuel distribution alone, the $\mathrm{CH}^{*}$ chemiluminescence distributions are considered next. Chemiluminescence is emitted from intermediate species in the flame front and its intensity is a measure for the heat release rate [21]. For the flames studied in this paper, the chemiluminescence from $\mathrm{CH}$ radicals was detected by an intensified CCD camera [7]. The line- 
of-sight integrated chemiluminescence distributions were Abel-inverted to yield a quasi-twodimensional distribution in the central vertical plane of the flames where the CARS measurements were performed [7]. Figure 10 displays these distributions for n-dodecane and nhexane. It is clearly seen that the flame shapes are different. For n-dodecane the flame zone is mainly located in a cone that extends from $(x=-10 \mathrm{~mm}, y=5 \mathrm{~mm})$ to $(x=-30 \mathrm{~mm}, \mathrm{y}=35 \mathrm{~mm})$. This region coincides largely with the spray distribution, i.e. the flame burns predominantly within the region of large droplet loading (see Figure 9). There is only very little heat release close to the flame axis within the inner recirculation zone. For the n-hexane flame, in contrast, the region of heat release is more distributed with significant combustion taking place in the inner recirculation zone. The second main area of heat release around $(\mathrm{x}=15-20 \mathrm{~mm}, \mathrm{y}=15 \mathrm{~mm})$ lies within the spray. However, there is also a broad region of heat release above $y=30 \mathrm{~mm}$ where no liquid fuel is present, but supposedly considerable amount of evaporated fuel. The temperature distributions are in qualitative agreement with the heat release distributions: intense heat release leads to an increase in temperature. The radial profiles in Figure 10b demonstrate this for the downstream location at $y=25 \mathrm{~mm}$. The large temperature gradients between $\mathrm{x}=-17 \mathrm{~mm}$ and -25 $\mathrm{mm}$ coincide with the regions of high heat release. Near the flame axis, the situation is more complex because the recirculation of hot combustion products also contributes to the temperature distribution. However, the higher temperature level of the n-hexane flame in this region compared to $n$-dodecane is certainly caused by the higher heat release rate.
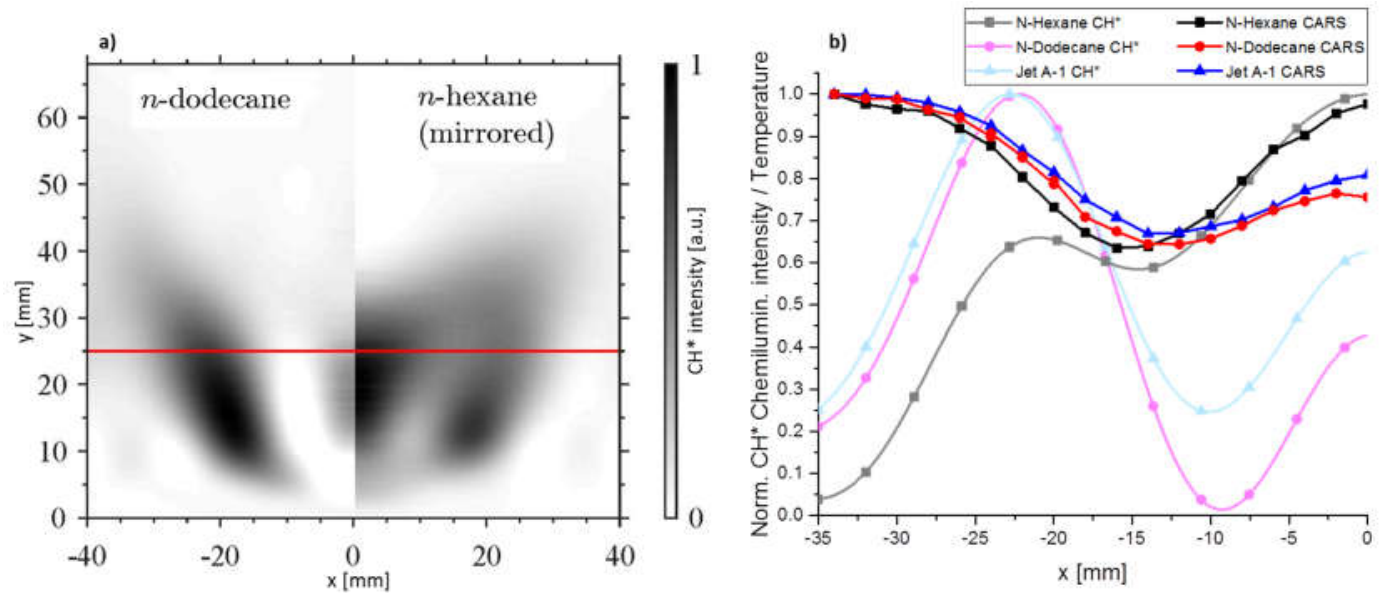

Figure 10. a) $\mathrm{CH}^{*}$ Chemiluminescence [8] $\mathrm{mm}$ for $\mathrm{n}$-dodecane and $\mathrm{n}$-hexane (mirrored); red line represents $\mathrm{y}=25 \mathrm{~mm}$; b) normalized $\mathrm{CH}^{*}$ chemiluminescence intensity compared to normalized $\mathrm{T}_{\text {mean }}$ measurements at $\mathrm{y}=25 \mathrm{~mm}$ for all fuels.

The temperature differences between the n-dodecane and n-hexane flames that are observed in the outer radial regions beyond $\mathrm{x}=-20 \mathrm{~mm}$ cannot unambiguously be explained by the experimental results. It is certainly a combination of heat release distribution, convective transport and heat loss by wall contact. It should also be considered that fuels with a large evaporation rate, such as n-hexane, enable a faster mixing of (evaporated) fuel and air. Therefore these flames burn on the average at leaner conditions than flames with small evaporation rate, such as n-dodecane and Jet A-1. Consequently, peak temperatures from combustion at nearstoichiometric conditions occur more frequently in the n-dodecane and Jet A-1 flames compared to the n-hexane flame. 


\section{Conclusions}

Temperature measurements using SV-CARS were successfully performed in a gas turbine model combustor. Three fuels (n-hexane, n-dodecane and kerosene Jet A-1) were tested at $\Phi=0.8$ and atmospheric pressure to provide quantitative inputs for numerical model validation. The overall goal is to provide a detailed understanding of the combustion of liquid single-component hydrocarbons in order to optimize combustion performance of jet fuel blends. Temperature profiles were recorded at the axial positions $\mathrm{y}=15,25$ and $35 \mathrm{~mm}$ and underlined the different regions in the flame. Low values correspond to the inflow region where cold and burned gases were found. Higher values were found in the outer radial region where the outer recirculation zone was present. The shear layers between the inflow and the recirculation zones were characterized by large fluctuations of temperature and velocity. Differences were found between the n-hexane and n-dodecane flames. The short alkane showed higher temperatures in the inner recirculation zones and lower in the outer one due to different atomization and vaporization. The variation of the thermal state was determined by the pdfs. Histograms generated by single shot measurements underlined the intermittency of the phenomena. Steady values with a mono-modal and high temperature distribution were found at the flame edges where mostly exhaust gas was present. However, in the flame core, strong temperature fluctuations were found showing bimodal distributions. This behavior can be caused by shear layer fluctuations or randomly flapping flame fronts. Temperature measurements were in a good agreement with previously performed Mie scattering and $\mathrm{CH}^{*}$ chemiluminescence measurements. High droplet loading corresponded to low temperatures and vice-versa. Differences were found between the light (n-hexane) and heavy (n-dodecane, Jet A-1) fuels in terms of atomization and vaporization rate. The $\mathrm{CH}^{*}$ chemiluminescence and temperature distributions were in qualitative agreement showing that the intense heat release in the flame zone led to an increase in temperature. It was observed that $\mathrm{n}$ dodecane burned predominantly within the spray region while n-hexane showed also considerable heat release in the inner recirculation zone and further downstream where the spray was already vaporized.

\section{Acknowledgments}

The authors thank Dr. Rainer Lückerath for his support with the CARS data evaluation. The financial support from the German Federal Ministry for Economic Affair and Energy through the InnoTreib project is gratefully acknowledged. The authors are also thankful for funding within the ECLIF project.

\section{References}

[1] T. Edwards, C. Moses, T. Dryer, Evaluation of Combustion Performances of Alternative Aviation Fuels, AIAA 2010-7155 (2010).

[2] J. S. Heyne, M. Colket, M. Gupta, A. Jardines, J. Moder, J. T. Edwards, W. M. Roquemore, C. Li, M. Rumizen, Year 2 of the National Jet Fuel Combustion Program: Moving Towards a Streamlined Alternative Jet Fuels Certification Program, AIAA 2017-0145 (2017).

[3] S. Blakey, L. Rye, C. W. Wilson, Aviation Gas Turbine Alternative Fuels: a Review, Proc. Comb. Inst. 35 (2015) 117-144.

[4] E. Corporan, T. Edwards, S. Stouffer, T. Hendershott, M. DeWitt, C. Klingshirn, Z. West, C. Bruening, Impact of Fuel Properties on Combustor Performance: Operability and Emission Characteristics, AIAA 2017-0380 (2017). 
[5] J. Grohmann, W. O'Loughlin, W. Meier, M. Aigner, Comparison of the Characteristics of Liquid Single-Component Fuels in a Gas Turbine Model Combustor, Proc. of ASME Turbo Expo (2016), GT2016-56177.

[6] G. Eckel, J. Grohmann, M. Rachner, P. LeClercq, M. Aigner, LES of a Generic SwirlStabilized Spray Burner with Detailed Chemistry, Proc. of the $9^{\text {th }}$ US National Comb. Meeting (2015), No. 1B17.

[7] J. Grohmann, B. Rauch, T. Kathrotia, W. Meier, M. Aigner, Investigation of Differences in Lean Blowout of Liquid Single-Component Fuels in a Gas Turbine Model Combustor, AIAA 2016-4647 (2016).

[8] J. Grohmann, W. Meier, M. Aigner, Gas Turbine Model Combustor Emission of Liquid Single-Component Fuels, Proc. of ASME Turbo Expo (2017), GT2017-63182.

[9] P. D. Maker, R. W. Terhune, Study of Optical Effects Due to an Induced Polarization Third Order in the Electric Field Strength, Applied Physics Letters 23 (1973) 240-242

[10] A. C. Eckbreth, Laser Diagnostics for Combustion Temperature and Species, second ed., Taylor \& Francis, 2002, pp. 281-366.

[11] K. P. Geigle, Y. Schneider-Kühnle, M. S. Tsurikov, R. Hadef, R. Lückerath, V. Krüger, W. Stricker, M. Aigner, Investigation of Laminar Flames for Soot Model Validation Using SVCARS and LII, Proc. Comb. Inst. 30 (2005) 1645-1653.

[12] K. P. Geigle, M. Köhler, W. O’Loughlin, W. Meier, Investigation of Soot Formation in Pressurized Swirl Flames by Laser Measurements of Temperature, Flame Structures and Soot Concentration, Proc. Comb. Inst. 35 (2015) 3373-3380.

[13] E. C. A. Gallo, L. M. L. Cantu, A. D. Cutler, M. Rahimi, H. Chelliah, WIDECARS Measurements of Major Species Concentration and Temperature in an Air-Ethylene Flame, AIAA 2014-2525 (2014).

[14] E. C. A. Gallo, L. M. L. Cantu, A. D. Cutler, R. D. Rockwell, C. Goyne, J. McDaniel, WIDECARS Measurements of a Premixed Ethylene-Air Flame in Dual-Mode Scramjet, AIAA 2016-1763 (2016).

[15] R. Lückerath, M. Woyde, W. Meier, W. Stricker, U. Schnell, H. C. Magel, J. Görres, H. Spliethoff, H. Maier, Comparison of Coherent Anti-Stokes Raman-Scattering Thermometry with Thermocouple Measurements and Model Prediction in Both Natural-Gas and Coal-Dust Flames, Applied Optics 34 (1995) 3303-3312.

[16] K. P. Geigle, Y. Schneider-Kühnle, M. S. Tsurikov, R. Hadef, R. Lückerath, V. Krueger, W. Stricker, M. Aigner, Investigation of Laminar Flames for Soot Model validation Using SVCARS and LII, Proc. Comb. Inst. 30 (2005) 1645-1653.

[17] M. Colket, T. Edwards, S. Williams, N. P. Cernansky, D. L. Miller, F. N. Engolfopoulos, P. Lindtstet, K. Seshadri, F. Dryer, C. K. Law, D. Friend, D. B. Lenhert, H. Pitsch, A. Sarofim, M. Smoke, W. Tsang, Development of an Experimental Database and Kinetic Models for Surrogate Jet Fuels, AIAA 2007-0770 (2007).

[18] T. Edwards, L. Q. Maurice, Surrogate Mixtures to Represent Complex Aviation and Rocket Fuels, J. of Prop. and Power 17 (2001) 1157-1163.

[19] M. A. Gregor, F. Seffrin, F. Fuest, D. Geyer, A. Dreizler, Multi-Scalar Measurements in a Premixed Swirl Burner Using 1D Raman/Rayleigh Scattering, Proc. Comb. Inst. 32 (2009) 1739-1746.

[20] W. Meier, C. Dem, C.M. Arndt, Mixing and reaction Progress in a Confined Swirl Flame Undergoing Thermo-Acoustic Oscillations Studied with Laser Raman Scattering, Exp. Thermal and Fluid Science 73 (2016) 71-78. 
[21] B. Prabasena, M. Röder, T. Kathrotia, U. Riedel, T. Dreier, C. Schulz, Strain Rate and Fuel Composition Dependence of Chemiluminescent Species Profiles in Non-Premixed Counterflow Flames: Comparison with Model Results, Appl. Phys. B 107 (2012) 561-569. 